Pacific Journal of Mathematics

THE CARATHÉODORY METRIC AND HOLOMORPHIC MAPS
ON A CLASS OF WEAKLY PSEUDOCONVEX DOMAINS Michael RANGE 


\title{
THE CARATHÉODORY METRIC AND HOLOMORPHIC MAPS ON A CLASS OF WEAKLY PSEUDOCONVEX DOMAINS
}

\author{
R. Michael RANGe
}

\begin{abstract}
The boundary behavior of proper holomorphic maps between two smoothly bounded pseudoconvex domains in $C^{n}$ is studied by means of the Caratheodory metric. The Hölder continuity of such maps is proved in case the image domain satisfies some technical conditions; these are satisfied, for example, by strictly pseudoconvex domains and convex domains with real analytic boundary.
\end{abstract}

In recent years it has become clear that pseudoconvex domains with smooth boundary may exhibit rather pathological behavior in the absence of strict pseudoconvexity (cf. the examples of Kohn and L. Nirenberg [13] and Diederich and Fornaess [4]). Therefore it might be of interest to consider conditions weaker than strict pseudoconvexity and to extend classical results to more general settings.

Investigations related to Hölder estimates for solutions of the $\bar{\partial}$-equation (cf. Range [18]) have led the author to introduce a technical refinement of the following classical condition (cf. Behnke and Thullen [1], p. 29): The domain $D$ is called totally pseudoconvex at $P \in \partial D$ if there is an analytic hypersurface $M_{P}$ in a neighborhood $U$ of $P$, such that $M_{C} \cap \bar{D}=\{P\}$. The refinement involves two parts. First, there should be supporting analytic hypersurfaces $M_{\zeta}$ for all points $\zeta \in \partial D$ near $P$, and $M_{\zeta}$ should depend smoothly on $\zeta$. Next, in order to obtain estimates of some sort, one needs finite order contact between $M_{\zeta}$ and $\partial D$ at $\zeta$. The resulting condition is called uniform total pseudoconvexity of finite order (cf. Definition 1.8 for the precise formulation). Simple examples of domains which satisfy this condition at every boundary point are strictly pseudoconvex domains and convex domains with real analytic boundary.

In this paper we prove the following generalization of a classical result.

MaIn Theorem. Let $D_{1}$ and $D_{2}$ be bounded domains in $C^{n}$ with smooth boundary. Assume that $D_{2}$ is uniformly totally pseudoconvex of finite order at every point $P \in \partial D_{2}$, and that $\bar{D}_{2}$ has a Stein neighborhood basis. ${ }^{1}$ Then there is $\alpha>0$, such that every proper

1 Theorem 2.2 and, as a consequence, the Main Theorem, are valid without assuming the existence of a Stein neighborhood basis, provided one assumes high differentiability 
holomorphic map $F: D_{1} \rightarrow D_{2}$ is Hölder continuous of order $\alpha$; in particular, $F$ extends continuously to $\bar{D}_{1}$.

ADDED IN PROOF. The Main Theorem holds without requiring the existence of a Stein neighborhood basis for $D_{2}$. The necessary modifications for the proof are sketched in footnote 1 .

For $D_{2}$ strictly pseudoconvex (plus a mild restriction for $D_{1}$ ) the Main Theorem was proved by Henkin [9] and, independently, by Pinchuk [16]; a somewhat weaker result was obtained by Vormoor [20]. Based on these results, the author [17] proved the Main Theorem for biholomorphic maps between domains with piecewise smooth strictly pseudoconvex boundary. By a different method, Fefferman [5] proved that a biholomorphic map between strictly pseudoconvex domains with $C^{\infty}$ boundary extends as a $C^{\infty}$ diffeomorphism $\bar{D}_{1} \rightarrow \bar{D}_{2}$.

The proof of the Main Theorem uses the argument of Henkin and Pinchuk. The main analytic tool is an estimate for the Carathéodory metric of the image domain $D_{2}$. For strictly pseudoconvex domains such an estimate was obtained by Henkin and Pinchuk, and also by Graham [6], by approximating the domain by balls and using supremum norm estimates for $\bar{\partial}$. The proof given here is based on an explicit local construction, and the passage from local to global is handled by Hörmander's $L^{2}$ estimates for $\bar{\partial}$; in particular, one obtains a new proof for the strictly pseudoconvex case.

Briefly, this paper is organized as follows. In $\S 1$ we introduce the various notions of total pseudoconvexity and prove some basic results; in particular, we discuss the relationship with (Euclidean) convexity and the existence of peaking functions. The estimate for the Carathéodory metric is proved in $\S 2$. In $\S 3$ we combine a result of Diederich and Fornaess [3] and the estimates of $\S 2$ with the techniques of Henkin and Pinchuk to prove the Hölder continuity of proper holomorphic maps.

The results in $\S 1$ were, essentially, obtained in 1975 , but they have not been published in detail before. The author has lectured on several occasions about different versions of these results in the

of the boundary. In order to see this, one observes that in the proof of Theorem 2.2 the $\bar{\partial}$-closed $(0,1)$ forms $\alpha_{j}, 1 \leqq j \leqq n$, which are defined on $D_{\varepsilon}$, satisfy, for each $k \in N$, an estimate $\left\|\alpha_{j}\right\|_{C_{0,1}^{k}(\bar{D}) \leqq \gamma_{k}}\left\|\alpha_{j}\right\|_{L_{0,1}^{\infty}\left(L_{\varepsilon}\right)}$, where $0<\gamma_{k}<\infty$. By Kohn's global regularity result ([12], Theorem 3.19) and Sobolev's Lemma, for sufficiently large $k$ there is a bounded solution operator $T_{k}: C_{0.1}^{k} \bar{D} \cap \operatorname{ker} \bar{\partial} \rightarrow C^{1}(\bar{D})$ for $\bar{\partial}$. So $u_{j}=T_{k}\left(\alpha_{j}\right)$ satisfies $\bar{\partial} u_{j}=\alpha_{j}$ and $\left\|u_{j}\right\|_{C^{1}(\bar{D})} \leqq B_{k} \|_{L_{0,1}^{\infty}\left(D_{\varepsilon}\right)}$ for some constant $B_{k}$; this estimate is sufficient to complete the proof of (2.2). 
context of Hölder estimates for $\bar{\partial}$, notably at the 1975 Seminar on Spaces of Analytic Functions in Kristiansand, Norway, and at the 1975 AMS Summer Institute on Several Complex Variables in Williamstown, Massachusetts (cf. [18]). Initially, uniform total pseudoconvexity of finite order was formulated in terms of special coordinate systems which are now relegated to a technical device; the version adopted here, which emphasizes the supporting analytic hypersurface, seems the more natural one. The problem of finding a tractable characterization of total pseudoconvexity in terms of local invariants of the boundary remains open; its solution should contribute to a better understanding of pseudoconvexity.

Notations. For $x \in \boldsymbol{R}^{n}$ and $E \subset \boldsymbol{R}^{n}, d(x, E)$ denotes the Euclidean distance from $x$ to $E$. For $a \in C^{n}$, the components of $a$ are denoted by $a_{1}, \cdots, a_{n}$; we sometimes write $a=\left(a^{\prime}, a_{n}\right)$, where $a^{\prime}=$ $\left(a_{1}, \cdots, a_{n-1}\right) \in C^{n-1}$; the Euclidean norm $\left(\sum_{i=1}^{n} a_{i} \bar{a}_{i}\right)^{1 / 2}$ is denoted by $|a|$. For $P \in C^{n}$ and $c>0, B(P, c)$ denotes the open ball in $C^{n}$ with center $P$ and radius $c ; \Delta$ denotes the open unit disc in $C$ with center 0 .

For a $C^{1}$ function $f$ in a neighborhood of $P \in C^{n}, \partial f(P)$ denotes the $(1,0)$ form $\partial f=\sum_{i=1}^{n} \partial f / \partial z_{i}(P) d z_{i}$. The natural pairing between a cotangent vector $\alpha$ at $P$ and a tangent vector $v$ at $P$ is denoted by $\langle\alpha, v\rangle$; in particular, $\langle\partial f(P), v\rangle=\sum_{i=1}^{n} \partial f / \partial z_{i}(P) v_{i}$.

A domain $D \subset C^{n}$ has a $C^{k}$ boundary at $P \in \partial D, 1 \leqq k \leqq \infty$, if there is a $C^{k}$ function $r: U \rightarrow \boldsymbol{R}$ defined on a neighborhood $U$ of $P$, such that $d r(P) \neq 0$ and $D \cap U=\{z \in U: r(z)<0\}$; a function $r$ satisfying these conditions is called a defining function for $D$ at $P$. The real tangent space of $\partial D$ at $P$ is denoted by $T_{P}(\partial D)$, and the complex tangent space $T_{P}(\partial D) \cap \sqrt{-1} T_{P}(\partial D)$ is denoted by $H_{P}(\partial D)$; for any defining function $r, H_{p}(\partial D)=\left\{v \in C^{n}:\langle\partial r(P), v\rangle=0\right\}$.

In order to avoid the use of many constants, we adopt the following convention: If $A(x)$ and $B(x)$ denote expressions which depend on a variable $x \in \boldsymbol{R}^{l}, A(x) \lesssim B(x)$ means that there is a constant $K, 0<K<\infty$, such that $|A(x)| \leqq K|B(x)|$ for all $x$ under consideration; $A(x) \sim B(x)$ is equivalent to $A(x) \lesssim B(x)$ and $B(x) \lesssim$ $A(x)$.

1. Total pseudoconvexity. We first discuss some properties of the simple point version of total pseudoconvexity which may be of independent interest.

Definition 1.1. A domain $D$ in $C^{n}$ is called totally pseudoconvex at the point $P \in \partial D$ if there is a nonsingular analytic hypersurface $M$ in a neighborhood $U$ of $P$, such that $M \cap \bar{D} \cap U=\{P\} . \quad M$ is called a supporting analytic hypersurface for $D$ at $P$. 
From now on we will assume that $D$ has a $C^{1}$ boundary at $P$; the coordinates of $C^{n}$ and the defining function $r$ for $D$ are chosen so that $P=0, H_{0}(\partial D)=\left\{z \in C^{n}: z_{n}=0\right\}$ and $\partial r(0)=d z_{n}$. If $M$ is a supporting analytic hypersurface for $D$ at 0 , the tangent space of $M$ at 0 coincides with $H_{0}(\partial D)$; so, near $0 \in C^{n}, M$ can be described as the graph of a holomorphic function $g$ defined in a neighborhood $U^{\prime}$ of $0 \in C^{n-1}: M \cap\left(U^{\prime} \times C\right)=\left\{\left(z^{\prime}, z_{n}\right) \in U^{\prime} \times C: z_{n}=g\left(z^{\prime}\right)\right\}$. To say that $D$ is totally pseudoconvex at $0 \in \partial D$ is therefore equivalent to the following: There is a holomorphic function $g$ on $U^{\prime}$, with $g(0)=$ 0 , such that $r\left(z^{\prime}, g\left(z^{\prime}\right)\right)>0$ for $z^{\prime} \neq 0$.

It will be convenient to linearize $M$ by a suitable holomorphic change of coordinates. Choose a holomorphic function $\phi$ on a neighborhood $U$ of 0 , such that $M \cap U=\{z \in U: \phi(z)=0\}$ and $d \phi(0)=d z_{n}$. Define $F: U \rightarrow C^{n}$ by $w=F(z)=\left(z^{\prime}, \phi(z)\right)$; the Jacobian matrix $F_{* 0}$ of $F$ at 0 is the identity matrix; hence, after shrinking $U, F: U \rightarrow$ $F(U)$ is biholomorphic, and

$$
F(U \cap M)=F(U) \cap\left\{w \in C^{n}: w_{n}=0\right\}=F(U) \cap H_{0}(\partial F(D \cap U)) .
$$

we thus have the following definition equivalent to Definition 1.1.

1.2. $D$ is totally pseudoconvex at $P \in \partial D$ if there is a holomorphic change of coordinates $w=w(z)$ in a neighborhood $U$ of $P$, such that

$$
w(\bar{D} \cap U) \cap H_{w(P)}(\partial w(D \cap U))=\{w(P)\} .
$$

So, geometrically, total pseudoconvexity is just the biholomorphic image of convexity in the directions of the complex tangent vectors. The following result shows that by relaxing the regularity of the coordinate change at $P$, one can achieve convexity also in the remaining tangential direction.

Proposition 1.3. Let $D$ be totally pseudoconvex at $P \in \partial D$. Then there are a neighborhood $U$ of $P$, a neighborhood $\Omega$ of $\bar{D} \cap U-\{P\}$, and a biholomorphic map $G: \Omega \rightarrow G(\Omega) \subset C^{n}$ with the following properties:

(a) $G$ extends continuously to $P$ and $G(P)=0$;

(b) $G(\bar{D} \cap U-\{P\}) \subset\left\{w \in C^{n}: \operatorname{Re} w_{n}>0\right\}$.

Proof. Choose the coordinates of $C^{n}, r$ and $F$ as before; $\rho=r \circ F^{-1}$ is a defining function for $F(D \cap U)$ at 0 , and $\partial_{w} \rho(0)=d w_{n}$. By assumption, there is $c>0$ such that $\rho\left(w^{\prime}, 0\right)>0$ for $0<\left|w^{\prime}\right|<c$. Therefore, by Taylor's theorem, if $|w|<c$ and $w^{\prime} \neq 0$,

$$
\begin{aligned}
\rho(w)= & \rho\left(w^{\prime}, w_{n}\right)=\rho\left(w^{\prime}, 0\right)+2 \operatorname{Re} \frac{\partial \rho}{\partial w_{n}}\left(w^{\prime}, 0\right) \cdot w_{n}+o\left(\left|w_{n}\right|\right) \\
& >2 \operatorname{Re} 1 \cdot w_{n}+o(1) \cdot\left|w_{n}\right| .
\end{aligned}
$$


Choose $U$ so small that $o(1) \cdot\left|w_{n}\right| \leqq\left|\operatorname{Re} w_{n}\right|+\left|\operatorname{Im} w_{n}\right|$ for $w \in F(U)$; if $\rho(w) \leqq 0$, one obtains

$$
0>2 \operatorname{Re} w_{n}-\left|\operatorname{Re} w_{n}\right|-\left|\operatorname{Im} w_{n}\right|,
$$

or

(1.4.) $\quad-\operatorname{Re} w_{n}>-\left|\operatorname{Im} w_{n}\right|$ for $w \in F(\bar{D} \cap U)$ with $w^{\prime} \neq 0$.

This shows that, on $F(\bar{D} \cap U-\{P\}),-w_{n}$ omits the nonpositive real axis $\boldsymbol{R}^{-}$. If one chooses that branch of the square root defined on $\boldsymbol{C}-\boldsymbol{R}^{-}$which satisfies $\sqrt{1}=1, \sqrt{-w_{n}}$ is holomorphic on $\boldsymbol{C}-\boldsymbol{R}^{-}$ and, by 1.4, satisfies $\operatorname{Re} \sqrt{-w_{n}}>0$ for $w \in F(\bar{D} \cap U-\{P\})$, and hence for $w \in F(\Omega)$, where $\Omega$ is some neighborhood of $\bar{D} \cap U-\{P\}$. If $S(w)=\left(w^{\prime}, \sqrt{-w_{n}}\right)$, the map $G=S \circ F$ is biholomorphic on $\Omega$ and satisfies (a) and (b).

REMARK. As the proof shows, the singularity of $G$ at $P$ is quite simple. It is not known whether one can choose $G$ holomorphic at $P$, so that $1.3(\mathrm{~b})$ still holds. ${ }^{2}$

CoRollary 1.5. Let $D$ be totally pseudoconvex at $P \in \partial D$. Then there is a neighborhood $U$ of $P$ such that $P$ is a peak point for the uniform algebra $A(D \cap U)$.

Proof. The function $h(z)=\exp \left[-G_{n}(z)\right]$ is in $A(D \cap U)$ and peaks at $P$.

By using Kohn's global regularity result for $\bar{\partial}[12]$ and standard techniques (cf. Pflug [15], or Hakim and Sibony [8]), one obtains the following global version of $\mathbf{1 . 5}$.

Corollary 1.6. Suppose $D$ is a bounded pseudoconvex domain with smooth boundary. If $D$ is totally pseudoconvex at $P$, then $P$ is a peak point for the uniform algebra $A(D)$.

Furthermore, by a variation of the proof of a result of Rossi ([19], Theorem 5.13), Corollary 1.5 implies:

CoRollary 1.7. Suppose $D$ has a $C^{2}$ boundary near $P$ and $D$ is totally pseudoconvex at $P$. Then $P$ is a limit point of strictly pseudoconvex boundary points of $D$.

For the details of the simple modification required, see, for example, [8].

2 T. Bloom recently found an example for which there is no such map $G$ holomorphic at $P$. (cf. Duke Math. J. 45 (1978), 133-148.) 
We now come to the parametrized version of total pseudoconvexity.

Definition 1.8. Let $D$ have a $C^{1}$ boundary at $P \in \partial D$, and let $r$ be a defining function for $D . D$ is uniformly totally pseudoconvex at $P$ if there are positive constants $\delta, c$ and $a C^{1}-m a p \phi: \partial D \cap$ $B(P, \delta) \times B(P, 2 \delta) \rightarrow C$, such that, for all $\zeta \in \partial D \cap B(P, \delta)$ the following are satisfied:

(i) $\phi(\zeta, \cdot)$ is holomorphic on $B(\zeta, \delta)$;

(ii) $\phi(\zeta, \zeta)=0$ and $\left.d_{z} \phi\right|_{z=\zeta} \neq 0$;

(iii) $\quad r(z)>0$ for all $z$ with $\phi(\zeta, z)=0$ and $0<|z-\zeta|<c$.

Clearly Definition 1.8 implies that $D$ is totally pseudoconvex at all points $\zeta \in \partial D$ near $P$; the supporting analytic hypersurface for $D$ at $\zeta$ is given by $M_{\zeta}=\{z: \phi(\zeta, z)=0\}$.

For $\zeta \in \partial D$, we denote by $\pi_{\zeta}$ the orthogonal projection $T_{\zeta} C^{n} \rightarrow$ $H_{\zeta}(\partial D)$.

1.8 (Continued). $D$ is uniformly totally pseudoconvex of finite order at $P$ if, in addition to (i), (ii), (iii), there are $m \in N$ and $\gamma>0$, such that

(iv) $r(z) \geqq \gamma\left|\pi_{\zeta}(z-\zeta)\right|^{m}$ for all $z \in B(\zeta, c)$ with $\phi(\zeta, z)=0$.

REMARK 1.9. Definition 1.8 is independent of the choice of holomorphic coordinates in a neighborhood of $P$ and of the particular defining function $r$ which appears in (iii) and (iv). The smallest integer $m$ for which (iv) holds with some constants $\gamma$ and $c$ for all $\zeta \in \partial D$ in a neighborhood of $P$ is called the order of $\partial D$ at $P$. Note that if $D \subset C^{n}$ with $n>1$, one must have $m \geqq 2$.

By multiplying $r$ and $\phi$ by suitable nonzero functions of $\zeta$, one may further assume

$$
|\partial r(\zeta)|=1 \text { and } \partial r(\zeta)=\left.d_{z} \phi\right|_{z=\zeta} \text { for } \zeta \in \partial D \cap B(P, \delta) \text {. }
$$

EXAMPLe 1.10. A bounded domain $D \subset \boldsymbol{R}^{d}$ with a $C^{1}$ boundary is called totally convex if for each $P \in \partial D$ the tangent space $T_{p}(\partial D)$ intersects $\bar{D}$ only at $P$. If $D \subset C^{n}$ is totally convex, then $D$ is uniformly totally pseudoconvex at all points $P \in \partial D$; in fact, if $r$ is a defining function for $D$ in some neighborhood $U$ of $\partial D$, the function $\phi(\zeta, z)=\sum_{i=1}^{n} \partial r / \partial \zeta_{i}(\zeta)\left(z_{i}-\zeta_{i}\right)$ satisfies $1.8(\mathrm{i})$-(iii).

Example 1.11. Let $D$ be strictly Levi pseudoconvex at $P \in \partial D$, i.e., if $r$ is a $C^{2}$ defining function for $D$ near $P$, the Levi form

$$
L(r ; P, v)=\sum_{i, j=1}^{n} \frac{\partial^{2} r}{\partial \zeta_{i} \partial \bar{\zeta}_{j}}(P) v_{i} \bar{v}_{j}
$$


satisfies, with some $\gamma>0$,

$$
L(r ; P, v) \geqq \gamma|v|^{2} \text { for } v \in H_{p}(\partial D) .
$$

By continuity, it follows that $L(r ; \zeta, v) \geqq \gamma / 2|v|^{2}$ for $v \in H_{\zeta}(\partial D)$ and $\zeta \in \partial D$ near $P$. It is classical that in this case $D$ is uniformly totally pseudoconvex of finite order 2 at $P$; the function $\phi$ is given by

$$
\phi(\zeta, z)=\sum_{i=1}^{n} \frac{\partial r}{\partial \zeta_{i}}(\zeta)\left(z_{i}-\zeta_{i}\right)+\frac{1}{2} \sum_{i, j=1}^{n} \frac{\partial^{2} r}{\partial \zeta_{i} \partial \zeta_{j}}(\zeta)\left(z_{i}-\zeta_{i}\right)\left(z_{j}-\zeta_{j}\right) .
$$

Typically, this is proved by choosing $r$ strictly plurisubharmonic (cf. Gunning and Rossi [7], Chapter IXB); however, it is easy to obtain this result by just using any defining function $r$, as follows. By Taylor's expansion,

$$
r(z)=r(\zeta)+2 \operatorname{Re} \phi(\zeta, z)+L(r ; \zeta, z-\zeta)+o\left(|z-\zeta|^{2}\right) ;
$$

fix $\zeta \in \partial D$; for $z$ with $\phi(\zeta, z)=0$ one has $z-\zeta=\pi_{\zeta}(z-\zeta)+o(|z-\zeta|)$; therefore, for some $c>0$, one obtains

$$
\begin{aligned}
r(z) & =L(r ; \zeta, z-\zeta)+o\left(|z-\zeta|^{2}\right) \geqq 1 / 2 L\left(r ; \zeta, \pi_{\zeta}(z-\zeta)\right) \\
& \geqq \gamma / 4\left|\pi_{\zeta}(z-\zeta)\right|^{2} \text { for all } z \in B(\zeta, c) \text { with } \phi(\zeta, z)=0 .
\end{aligned}
$$

If $D$ is not strictly pseudoconvex at $P$, it is usually condition 1.8 (iv) which is hardest to verify. Even though one may be able to obtain for each $\zeta \in \partial D$ near $P$ an estimate $r(z) \geqq \gamma_{\zeta}\left|\pi_{\zeta}(z-\zeta)\right|^{m_{\zeta}}$ for $z \in B\left(\zeta, c_{\zeta}\right)$ with $\dot{\phi}(\zeta, z)=0$, there remains the nontrivial problem of choosing $\gamma_{\zeta}, c_{\zeta}, m_{\zeta}$ independently of $\zeta$. As an example, consider, for $m>2$, even, the domain $B_{m}=\left\{\left|z_{1}\right|^{2}+\left|z_{2}\right|^{m}<1\right\}$ with defining function $r_{m}(z)=\left|z_{1}\right|^{2}+\left|z_{2}\right|^{m}-1$. $B_{m}$ is totally convex, and at points $\zeta=\left(\zeta_{1}, \zeta_{2}\right) \in \partial B_{m}$ with $\zeta_{2} \neq 0$ it is strictly pseudoconvex. If $\phi(\zeta, z)=$ $\left\langle\partial r_{m}(\zeta), z-\zeta\right\rangle$, one obtains the following estimates for $\zeta \in \partial B_{m}$ and $z \in B(\zeta, 1)$ with $\phi(\zeta, z)=0$ :

$$
r_{m}(z) \geqq\left\{\begin{array}{l}
\gamma_{\zeta}\left|\pi_{\zeta}(z-\zeta)\right|^{2} \geqq \gamma_{\zeta}\left|\pi_{\zeta}(z-\zeta)\right|^{m}, \quad \text { if } \zeta_{2} \neq 0 ; \\
1 \cdot\left|\pi_{\zeta}(z-\zeta)\right|^{m}, \quad \text { if } \zeta_{2}=0 .
\end{array}\right.
$$

Here, for $\zeta_{2} \neq 0, \gamma_{5}>0$ is, essentially, the eigenvalue of the Levi form; since $\gamma_{5} \rightarrow 0$ as $\zeta_{2} \rightarrow 0$, the constants one obtains by the "obvious" point estimates do not depend continuously on $\zeta$ at nonstrictly pseudoconvex boundary points. Nevertheless, as pointed out in [18], one can show that $B_{m}$ is uniformly totally pseudoconvex of finite order $m$.

More generally; one has the following result.

Proposition 1.12. Let $D$ be a bounded domain in $C^{n}$ with real analytic boundary. Suppose $D$ is uniformly totally pseudoconvex 
at every point $P \in \partial D$, and that the function $\phi(\zeta, z)$ given by 1.8 can be chosen real analytic in $(\zeta, z)$. Then $D$ is uniformly totally pseudoconvex of finite order at every $P \in \partial D$.

CoROllaRY 1.13. Let $D$ be a bounded convex domain in $C^{n}$ with real analytic boundary. Then $D$ is uniformly totally pseudoconvex of finite order at every $P \in \partial D$.

To prove the corollary, observe that the hypotheses imply that $D$ is totally convex; the conclusion then follows by 1.10 and the proposition.

In order to prove 1.12 we first introduce the parametrized version of the coordinate system given by 1.2; this will be used in $\S 2$ as well.

Thus, suppose $D$ is uniformly totally pseudoconvex at $P$, and let $U=B(P, \delta), c, r(z)$, and $\phi(\zeta, z)$ be as in 1.8, so that (i), (ii), (iii), and $(\mathrm{v})$ are satisfied. Choose smooth orthonormal sections $E^{(1)}(\zeta)$, $\cdots, E^{(n-1)}(\zeta)$ of the holomorphic tangent bundle $H(\partial D)$ over $\partial D \cap U$. For $\zeta \in \partial D \cap U$ define the holomorphic map $z \rightarrow w=F_{\zeta}(z)$ by

$$
w_{\nu}=\sum_{i=1}^{n} \bar{E}_{i}^{(\nu)}(\zeta)\left(z_{i}-\zeta_{i}\right), \nu=1, \cdots, n-1 \text {, and } w_{n}=\phi(\zeta, z) \text {. }
$$

The Jacobian matrix $\left(F_{\zeta}\right)_{* \zeta}$ has rows $\bar{E}^{(1)}(\zeta), \cdots, \bar{E}^{(n-1)}(\zeta),\left(\partial \dot{\phi} / \partial z_{1}(\zeta, \zeta)\right.$, $\left.\cdots, \partial \phi / \partial z_{n}(\zeta, \zeta)\right)$, and so it is unitary, by (v). After shrinking the neighborhood $U$ of $P$, one may choose $c>0, d>0$ so small that $F_{\zeta}$ maps $B(\zeta, c)$ biholomorphically onto the neighborhood $F_{\zeta}(B(\zeta, c)) \supset$ $B(0, d)$ of 0 in $C^{n}$ for all $\zeta \in \partial D \cap U$. Also, one may assume that $F_{\zeta}$ and $F_{\zeta}^{-1}$ have uniformly bounded Jacobian matrices; hence there are positive constants $A_{1}, A_{2}$ such that

$$
\begin{gathered}
A_{1}\left|z-z^{*}\right| \leqq\left|F_{\zeta}(z)-F_{\zeta}\left(z^{*}\right)\right| \leqq A_{2}\left|z-z^{*}\right| \\
\quad \text { for } z, z^{*} \in B(\zeta, c) .
\end{gathered}
$$

The analytic hypersurface $\{z \in B(\zeta, c): \phi(\zeta, z)=0\}$ is mapped by $F_{\zeta}$ biholomorphically into $\left\{w \in C^{n}: w_{n}=0\right\}$. The function $\rho_{\zeta}=r \circ \boldsymbol{F}_{\zeta}^{-1}$ is a defining function for $F_{\zeta}(D \cap B(\zeta, c))$; a calculation shows

$$
\partial_{w} \rho_{\zeta}(0)=d w_{n} \text {. }
$$

The conditions (iii) and (iv) are, respectively, equivalent to

(iii bis)

$$
\begin{aligned}
& \rho_{\zeta}\left(w^{\prime}, o\right)>0 \text { for } 0<\left|w^{\prime}\right|<d ; \\
& \rho_{\zeta}\left(w^{\prime}, o\right) \geqq \gamma\left|w^{\prime}\right|^{m} \text { for } 0 \leqq\left|w^{\prime}\right|<d .
\end{aligned}
$$

(iv bis)

Proof of Proposition 1.12. By assumption, the functions $r$ and $\dot{\rho}$ may be chosen real analytic, which implies that the map $F_{\zeta}(z)$ 
constructed above may be chosen real analytic in $(\zeta, z)$. One thus obtains a nonegative real analytic function $R\left(\zeta, w^{\prime}\right)=\rho_{5}\left(w^{\prime}, o\right)$ defined on $\Omega=(\partial D \cap U) \times\left\{w^{\prime} \in C^{n-1}:\left|w^{\prime}\right|<d\right\}$. Let $Z=\left\{\left(\zeta, w^{\prime}\right) \in \Omega: R\left(\zeta, w^{\prime}\right)=\right.$ $0\}$; by (iii bis), $Z=\left\{\left(\zeta, w^{\prime}\right) \in \Omega: w^{\prime}=0\right\}$, and $d\left(\left(\zeta, w^{\prime}\right), Z\right)=\left|w^{\prime}\right|$. By a theorem of Lojasiewicz ([14], p. 124), given $U^{\prime} \subset \subset U$ and $0<d^{\prime}<d$, one can find a constant $\gamma>0$ and a positive integer $m$, such that

$$
R\left(\zeta, w^{\prime}\right) \geqq \gamma d\left(\left(\zeta, w^{\prime}\right), Z\right)^{m}=\gamma\left|w^{\prime}\right|^{m} \text { for } \zeta \in \partial D \cap U^{\prime} \text { and }\left|w^{\prime}\right| \leqq d^{\prime} .
$$

Thus (iv bis) holds.

Finally, by modifying the proof of Proposition 1.3, one obtains a more precise estimate for $\phi(\zeta, z)$.

Proposition 1.16. Suppose $D$ is uniformly totally pseudoconvex of finite order $m$ at the point $P \in \partial D$. Let $\phi(\zeta, z)$ satisfy $(\mathrm{i})-(\mathrm{v})$ in (1.8) for $\zeta \in \partial D \cap B(P, \delta)$. Then there are constants $A, c^{\sharp}>0$, such that

(vi) $|\dot{\phi}(\zeta, z)| \geqq A\left[d(z, \partial D)+|\operatorname{Im} \phi(\zeta, z)|+|\zeta-z|^{m}\right]$ for $\zeta \in \partial D \cap B(P, \delta)$ and $z \in \bar{D} \cap B\left(\zeta, c^{\sharp}\right)$.

Proof. Fix $\zeta \in \partial D \cap B(P, \delta)$ and introduce $\rho_{\zeta}=r \circ F_{\xi}^{-1}$ as above. Taylor's theorem, 1.15 and (iv bis) imply, for $|w|<d$,

$$
\begin{aligned}
\rho_{\xi}\left(w^{\prime}, w_{n}\right) & =\rho_{\xi}\left(w^{\prime}, 0\right)+2 \operatorname{Re}\left(\frac{\partial \rho_{\xi}}{\partial w_{n}}\left(w^{\prime}, 0\right) \cdot w_{n}\right)+o\left(\left|w_{n}\right|\right) \\
& \geqq 2 \operatorname{Re} w_{n}+\gamma\left|w^{\prime}\right|^{m}+o(1) \cdot\left|w_{n}\right|,
\end{aligned}
$$

where $o(1) \rightarrow 0$ as $w \rightarrow 0$, the convergence being uniform in $\zeta$, as $o(1)$ depends only on the modulus of continuity of the first order partial derivatives of $r \circ F_{\zeta}^{-1}$.

Since $m \geqq 2, \quad\left|w^{\prime}\right|^{m}=|w|^{m}+o(1) \cdot\left|w_{n}\right|$; thus, if $0<d^{\sharp}<d$ is chosen so small that the combined terms $o(1) \cdot\left|w_{n}\right|$ satisfy $o(1) \cdot\left|w_{n}\right| \leqq$ $\left|\operatorname{Re} w_{n}\right|+\left|\operatorname{Im} w_{n}\right|$ for $|w| \leqq d^{\sharp}$, one obtains

$$
-2 \operatorname{Re} w_{n}+\left|\operatorname{Re} w_{n}\right| \geqq-\rho_{5}(w)-\left|\operatorname{Im} w_{n}\right|+\gamma|w|^{m} \text { for }|w| \leqq d^{\sharp},
$$

and hence

$$
3\left|\operatorname{Re} w_{n}\right| \geqq-\rho_{\zeta}(w)-\left|\operatorname{Im} w_{n}\right|+\gamma|w|^{m} .
$$

Choose $c^{\sharp}$ so small that $F_{\zeta}\left(B\left(\zeta, c^{\sharp}\right)\right) \subset B\left(o, d^{\sharp}\right)$. If $z \in B\left(\zeta, c^{\sharp}\right)$, the last inequality and (1.14) imply

$$
3|\operatorname{Re} \phi(\zeta, z)| \geqq-r(z)-|\operatorname{Im} \phi(\zeta, z)|+\gamma A_{1}|z-\zeta|^{m} .
$$

Since $|r(z)| \gtrsim d(z, \partial D)$ for $z \in \bar{D}$ and $5|\phi|>3|\operatorname{Re} \phi|+2|\operatorname{Im} \phi|$, it follows that 


$$
|\phi(\zeta, z)| \gtrsim d(z, \partial D)+|\operatorname{Im} \phi(\zeta, z)|+\gamma A_{1}|z-\zeta|^{m}
$$

for $z \in \bar{D} \cap B\left(\zeta, c^{\sharp}\right)$.

2. The Caratheodory metric. The infinitesimal form $C_{D}$ of the Carathéodory metric on a domain $D$ in $C^{n}$ is defined as follows: for $z \in D$ and $v \in C^{n}$,

$$
C_{D}(z, v)=\sup \{|\langle\partial f(z), v\rangle|: f: D \longrightarrow \Delta, \text { holomorphic }\} \text {. }
$$

For a holomorphic map $F: D_{1} \rightarrow D_{2}$ one trivially obtains

$$
C_{D_{2}}\left(F(z), F_{*_{z}} v\right) \leqq C_{D_{1}}(z, v),
$$

where $F_{*_{z}}$ denotes the Jacobian matrix of $F$ at $z$. Furthermore, by restricting $f: D \rightarrow \Delta$ to the ball $B(z, d(z, \partial D)) \subset D$ and applying Cauchy's derivative estimates, one obtains

Lemma 2.1. The Carathéodory metric satisfies

$$
C_{D}(z, v) \leqq|v| d(z, \partial D)^{-1}
$$

for all $z \in D$ and $v \in C^{n}$.

The main result of this section is the following estimate from below for the Carathéodory metric.

TheOREM 2.2. Let $D$ be a bounded domain in $C^{n}$ with $C^{1}$ boundary, and let $r$ be a defining function for $D$ defined on a neighborhood of $\bar{D}$. Suppose that $D$ is uniformly totally pseudoconvex of finite order $m$ at every point $P \in \partial D$, and that $\bar{D}$ has a Stein neighborhood basis ${ }^{1}$. Then

$$
C_{D}(z, v) \gtrsim|v| d(z, \partial D)^{-1 / m}+|\langle\partial r(z), v\rangle| d(z, \partial D)^{-1}
$$

for $z \in D$ and $v \in C^{n}$.

REMARK 2.3. $C_{D}(z, v)$ may grow faster than $d(z, \partial D)^{-1 / m}$ for certain tangential vectors $v$, but in general, no better estimate is possible for all $v$. As an example, consider $D=\left\{z \in C^{3}:\left|z_{1}\right|^{2}+\left|z_{2}\right|^{2}+\right.$ $\left.\left|z_{3}\right|^{4}<1\right\}$ and $P=(1,0,0) \in \partial D$; one can show that $D$ is uniformly totally pseudoconvex of order 4 at $P$; for $v=(0,1,0)$ and $v^{*}=$ $(0,0,1) \in H_{P}(\partial D)$ one obtains $C_{D}(z, v) \sim d(z, \partial D)^{-1 / 2}$ and $C_{D}\left(z, v^{*}\right) \sim$ $d(z, \partial D)^{-1 / 4}$ as $z \rightarrow P$ along the inner normal to $\partial D$ at $P$.

The proof of Theorem 2.2 involves a technical local result which we state separately. First we define, for $\zeta \in \partial D, \delta>0$ and $\varepsilon>0$,

$$
\Omega(\zeta, \delta, \varepsilon)=(D \cap B(\zeta, \delta)) \cup\{z: \delta / 2<|z-\zeta|<\delta ; r(z)<\varepsilon\} .
$$


MaIN Lemma 2.4. Let $D$ be uniformly totally pseudoconvex of order $m$ at $P \in \partial D$. Then there are positive real numbers $\delta, \varepsilon, a, M$, such that the following holds.

For each $x \in D \cap B(P, \delta)$, if $\zeta_{x} \in \partial D \cap B(P, 2 \delta)$ is chosen so that $\left|x-\zeta_{x}\right|=d(x, \partial D)$, there are

(i) functions $h_{1}^{x}, \cdots, h_{n}^{x}$ defined and holomorphic on $\Omega\left(\zeta_{x}, \delta, \varepsilon\right)$,

(ii) an orthonormal basis $v_{1}^{x}, \cdots, v_{n}^{x}$ of $C^{n}$ with $v_{n}^{x}$ perpendicular to $H_{\zeta x}(\partial D)$,

which satisfy the following conditions:

(iii) $\left|h_{j}^{x}(z)\right| \leqq M$ for $z \in \Omega\left(\zeta_{x}, \delta, \varepsilon\right), j=1, \cdots, n$;

(iv) $\left|\left\langle\partial h_{j}^{x}(x), v_{j}^{x}\right\rangle\right| \gtrsim d(x, \partial D)^{-1 / m}$ for $d(x, \partial D)<a$ and $f=1, \cdots$, $n-1$

(v) $\left|\left\langle\partial h_{n}^{x}(x), v_{n}^{x}\right\rangle\right| \gtrsim d(x, \partial D)^{-1}$ for $d(x, \partial D)<a$.

We first show how the Main Lemma implies the theorem.

Proof of 2.2. Fix $P \in \partial D$; for $x \in D \cap B(P, \delta)$ let $h_{1}^{x}, \cdots, h_{n}^{x}$ be the functions given by the Main Lemma. The essential part of the proof involves replacing these functions by functions $H_{j}^{x}, j=1, \cdots, n$, which are holomorphic on $D$ and still satisfy properties (iii), (iv), and ( $v$ ) above.

Choose $\chi \in C^{\infty}(\boldsymbol{R})$ such that $0 \leqq \chi \leqq 1$ and

$$
\chi(t)=\left\{\begin{array}{l}
1 \text { for } t \leqq 5 \delta / 8 \\
0 \text { for } t \geqq 7 \delta / 8 ;
\end{array}\right.
$$

define, for $\zeta \in \boldsymbol{C}^{n}$, the function $\chi_{\zeta} \in C_{0}^{\infty}\left(\boldsymbol{C}^{n}\right)$ by

$$
\chi_{\xi}(z)=\chi(|z-\zeta|) \text {. }
$$

Now fix $x \in D \cap B(P, \delta)$ with $d(x, \partial D)<\delta / 2$; to simplify notation, we will omit the superscript $x$ in $h_{j}^{x}$ and $v_{j}^{x}$, and we set $\zeta=\zeta_{x}$; unless otherwise noted, the index $j$ runs from 1 to $n$.

Set $\alpha_{j}=\bar{\partial}\left(\chi_{\zeta} h_{j}\right)$ on $\Omega(\zeta, \delta, \varepsilon) ; \alpha_{j}$ extends trivially as a $\bar{\partial}$-closed $C_{0,1}^{\infty}$ - form to the domain $D_{\varepsilon}=D \cup\{z: r(z)<\varepsilon\}$. Choose a Stein domain $G$, such that $D \subset \subset G \subset D_{\varepsilon}$. By Hörmander [10], there are functions $u_{j} \in C^{\infty}(G)$, such that $\bar{\partial} u_{j}=\alpha_{j}$ and

$$
\left\|u_{j}\right\|_{L^{2}(G)} \lesssim\left\|\alpha_{j}\right\|_{L^{2}(G)} \lesssim \sup _{z \in \Omega(\zeta, \delta, \varepsilon)}\left|h_{j}(z)\right| \leqq M .
$$

By interior elliptic estimates for $\bar{\partial}$,

$$
\sup _{z \in D}\left|u_{j}(z)\right| \lesssim\left\|u_{j}\right\|_{L^{1}(G)}+\left\|\bar{\partial} u_{j}\right\|_{L^{\infty}(G)} \leqq K_{1} \cdot M .
$$

Define $H_{j}=\chi_{\xi} h_{j}-u_{j} ; H_{j}$ is holomorphic on $D$, and by 2.4 (iii) and (2.5) 


$$
\left|H_{j}(z)\right| \leqq\left(1+K_{1}\right) \cdot M=M^{\prime} \text { for } z \in D \text {. }
$$

Furthermore, observe that $u_{j}$ is holomorphic on $G \cap B(\zeta, 5 \delta / 8)$; since for $z \in D \cap B(\zeta, \delta / 2)$ and $w \notin G \cap B(\zeta, 5 \delta / 8),|z-w| \geqq \min (\delta / 8$, dist $\left.\left(D, C^{n}-G\right)\right)>0$, it follows that for some constant $K_{2}$,

$$
\left|\partial u_{j}(z)\right| \lesssim\left\|u_{j}\right\|_{L^{2}(G)} \leqq K_{2} \cdot M \text { for } z \in D \cap B(\zeta, \delta / 2) \text {. }
$$

From $\partial H_{j}(x)=\partial h_{j}(x)-\partial u_{j}(x)$ one thus obtains

$$
\left|\left\langle\partial H_{j}(x), v_{j}\right\rangle\right| \geqq\left|\left\langle\partial h_{j}(x), v_{j}\right\rangle\right|-K_{2} \cdot M,
$$

which implies that (iv) and (v) in 2.4 still hold with $H_{j}$ instead of $h_{j}$, provided $d(x, \partial D)<a^{\prime}$, where $0<a^{\prime} \leqq a$ is suitably chosen. Since $H_{j} / M^{\prime}: D \rightarrow \Delta$, one has $C_{D}\left(x, v_{j}\right) \geqq 1 / M^{\prime}\left|\left\langle\partial H_{j}(x), v_{j}\right\rangle\right|$; it follows that

$$
C_{D}\left(x, v_{j}\right) \gtrsim d(x, \partial D)^{-1 / m}, \quad j=1, \cdots, n-1,
$$

and

$$
C_{D}\left(x, v_{n}\right) \gtrsim d(x, \partial D)^{-1} \text {. }
$$

By 2.4 (ii), this implies

$$
C_{D}(x, v) \gtrsim|v| d(x, \partial D)^{-1 / m}+|\langle\partial r(x), v\rangle| d(x, \partial D)^{-1}
$$

for all $v \in C^{n}$; here $x$ is any point in $D \cap B(P, \delta)$ with $d(x, \partial D)<a^{\prime}$, and the constant implicit in $\gtrsim$ is independent of $x$. A standard compactness argument now shows that the above estimate holds for all $x \in D$.

Proof of the Main Lemma. The plan of the proof is as follows: one first constructs the required functions and vectors with respect to the coordinate system $w=F_{\zeta_{x}}(z)$ given in $\S 1$, and then one pulls back everything to the domain $D$.

We use the notation developed in $\S 1 . \quad \delta>0$ is chosen so small that for all $\zeta \in \partial D \cap B(P, 2 \delta)$ the biholomorphic map $F_{\zeta}$ is defined on $B(\zeta, \delta)$ and $\rho_{\zeta}=r \circ F_{\zeta}^{-1}$ satisfies (1.17) for $|w|<d^{\sharp}$, i.e.,

$$
-2 \operatorname{Re} w_{n}+\left|\operatorname{Re} w_{n}\right|+\left|\operatorname{Im} w_{n}\right| \geqq-\rho_{\zeta}(w)+\gamma|w|^{m} .
$$

By (1.14), if $\delta$ is chosen sufficiently small, there is $b>0$, such that

$$
F_{\xi}(\{z: \delta / 2<|z-\zeta|<\delta\}) \subset\left\{w: b<|w|<d^{\sharp}\right\} .
$$

Let $\varepsilon=\gamma b^{m} / 2$ and define

$$
R(\zeta)=\left\{w:|w|<d^{\sharp}, \rho_{\zeta}(w)<0\right\} \cup\left\{w: b<|w|<d^{\sharp}, \rho_{\zeta}(w)<\varepsilon\right\} ;
$$

observe that $F_{\zeta}(\Omega(\zeta, \delta, \varepsilon)) \subset R(\zeta)$. 
(2.6) shows that for $w \in R(\zeta)$ the function $w_{n}$ omits the nonnegative real axis; hence one can define a holomorphic branch of $w_{n}^{1 / m}$ on $R(\zeta)$. For $j=1, \cdots, n-1$ we define holomorphic functions $g_{j}$ on $R(\zeta)$ by $g_{j}(w)=w_{j} \cdot w_{n}^{-1 / m}$; then

$$
\left(\frac{\partial}{\partial w_{j}} g_{j}\right)(w)=w_{n}^{-1 / m}, \quad j=1, \cdots, n-1 \text {. }
$$

From (2.6) one obtains

$$
\begin{aligned}
& 4\left|w_{n}\right| \geqq \varepsilon \text { if } b<|w|<d^{\#} \text { and } \rho_{\xi}(w)<\varepsilon ; \\
& 4\left|w_{n}\right| \geqq \gamma|w|^{m} \geqq \gamma\left|w_{j}\right|^{m} \text { if }|w|<d^{\#} \text { and } \rho_{\xi}(w)<0 ;
\end{aligned}
$$

this implies that there is a constant $M$ such that

$$
\left|g_{j}(w)\right| \leqq M \text { for } w \in R(\zeta), \quad j=1, \cdots, n-1 .
$$

In order to define $g_{n}$ we modify the function $f(w)=\exp \left(-\sqrt{-w_{n}}\right)$ which was used in the proof of Corollary 1.5; $f$ is well defined and holomorphic on $R(\zeta),|f(w)|<1$ for $w \in R(\zeta)$ and $f(w) \rightarrow 1$ for $w \rightarrow 0$. Fix $y \in R(\zeta)$ and let $\varphi_{q}$ be the holomorphic automorphism of $\Delta$ which sends $q=f(y)$ to 0 and 1 to 1 . Since $\left|\varphi_{q}^{\prime}(q)\right|=\left(1-|q|^{2}\right)^{-1}$ and

$$
1-|q|=1-|f(y)|=1-\exp \left(-\operatorname{Re} \sqrt{-y_{n}}\right) \lesssim\left|\sqrt{y_{n}}\right|,
$$

it follows that

$$
\left|\varphi_{q}^{\prime}(q)\right| \gtrsim\left|y_{n}\right|^{-1 / 2}
$$

Therefore, if one defines $g_{n}^{y}=\varphi_{f(y)} \circ f$, one obtains, by the chain rule,

$$
\left|\frac{\partial g_{n}^{y}}{\partial w_{n}}(y)\right| \gtrsim\left|y_{n}\right|^{-1}
$$

and

$$
\left|g_{n}^{y}(w)\right|<1 \text { for } w \in R(\zeta) \text {. }
$$

Now fix $x \in D \cap B(P, \delta)$ and choose $\zeta_{x} \in \partial D \cap B(P, 2 \delta)$ such that $\left|x-\zeta_{x}\right|=d(x, \partial D)$. Let $y=F_{\zeta_{x}}(x)$, and set $h_{j}^{x}=g_{j} \circ F_{\zeta_{x}}$ for $j=1$, $\cdots, n-1, h_{n}^{x}=g_{n}^{y} \circ F_{\zeta_{x}}$. Then, by (2.8) and (2.10), conditions 2.4 (i) and (iii) are satisfied. From the explicit form of the matrix $\left(F_{\zeta}\right)_{* \zeta}$ (cf. the definition of $F_{\zeta}$ in $\S 1$ ), it follows that the vectors $v_{j}^{x}=$ $\left(F_{\zeta_{x}}^{-1}\right)_{* 0}\left(\partial / \partial w_{j}\right), j=1, \cdots, n$, satisfy 2.4 (ii). Let $t_{j}^{x}=\left(F_{\zeta_{x}}^{-1}\right)_{* y}\left(\partial / \partial w_{j}\right)$; then

$$
v_{j}^{x}=t_{j}^{x}+o\left(\left|x-\zeta_{x}\right|\right)=t_{j}^{x}+o(d(x, \partial D)),
$$

and therefore there is $K<\infty$, such that

$$
\left|\left\langle\partial h_{j}^{x}(x), v_{j}^{x}-t_{j}^{x}\right\rangle\right| \leqq K
$$


whenever $d(x, \partial D)$ is sufficiently small. Also, $y_{n}=\phi\left(x, \zeta_{x}\right)$ implies $\left|y_{n}\right| \lesssim\left|x-\zeta_{x}\right|=d(x, \partial D)$. Hence, there is $a>0$, such that for all $x \in D \cap B(P, \delta)$ with $d(x, \partial D)<a$ one obtains, by (2.7),

$$
\begin{aligned}
\left|\left\langle\partial h_{j}^{x}(x), v_{j}^{x}\right\rangle\right| & \geqq\left|\left\langle\partial h_{j}^{x}(x), t_{j}^{x}\right\rangle\right|-K=\left|\frac{\partial g_{j}}{\partial w_{j}}(y)\right|-K \\
& \gtrsim d(x, \partial D)^{-1 / m} \text { for } j=1, \cdots, n-1,
\end{aligned}
$$

and, by (2.9),

$$
\left|\left\langle\partial h_{n}^{x}(x), v_{n}^{x}\right\rangle\right| \geqq\left|\frac{\partial g_{n}^{y}}{\partial w_{n}}(y)\right|-K \gtrsim d(x, \partial D)^{-1} .
$$

This completes the proof of the Main Lemma.

\section{Proper holomorphic maps.}

Lemma 3.1. Let $D_{1}$ and $D_{2}$ be bounded pseudoconvex domains in $C^{n}$ with smooth boundary. Then there is a positive integer $l$ such that every proper holomorphic map $F: D_{1} \rightarrow D_{2}$ satisfies

$$
d\left(z, \partial D_{1}\right)^{l} \lesssim d\left(F(z), \partial D_{2}\right) \lesssim d\left(z, \partial D_{1}\right)^{1 / l}
$$

for all $z \in D_{1}$.

Proof. By a theorem of Diederich and Fornaess [3], there are continuous functions $\phi_{\nu}: \bar{D}_{\nu} \rightarrow \boldsymbol{R}, \nu=1,2$, with the following properties:

(i) $\varphi_{\nu} \mid D_{\nu}$ is smooth and plurisubharmonic;

(ii) $\varphi_{\nu} \mid D_{\nu}<0$ and $\varphi_{\nu} \mid \partial D_{\nu}=0$;

(iii) for some $l \in N,\left(\varphi_{\nu}\right)^{l}$ is smooth on $\bar{D}_{\nu}$. iii implies

$$
\left|\varphi_{\nu}(x)\right| \lesssim d\left(x, \partial D_{\nu}\right)^{1 / l} \text { for } x \in D_{\nu} .
$$

Let $\dot{\psi}_{1}=\varphi_{2} \circ F$. Since $F$ is proper, $\psi_{1}$ is continuous on $\bar{D}_{1}$, and it satisfies (i) and (ii) with respect to $D_{1}$.

In order to push forward $\varphi_{1}$, observe that $F: D_{1} \rightarrow D_{2}$ represents $D_{1}$ as a $\lambda$-sheeted branched analytic covering over $D_{2}$. Define $\psi_{2}$ on $D_{2}$ by

$$
\psi_{2}(w)=\max \left\{\varphi_{1}\left(z_{1}\right), \cdots, \varphi_{1}\left(z_{\lambda}\right)\right\},
$$

where $\left\{z_{1}, \cdots, z_{\lambda}\right\}=F^{-1}(w)$, counted with multiplicities. $\psi_{2}$ is continuous on $\bar{D}_{2}$ and plurisubharmonic on $D_{2}$ (cf. [16], p. 646); also, $\psi_{2} \mid D_{2}<0$ and $\psi_{2} \mid \partial D_{2}=0$.

The classical normal derivative lemma [11], also known as Hopf lemma (cf. [2]), implies

$$
\left|\psi_{\nu}(x)\right| \gtrsim d\left(x, \partial D_{\nu}\right) \text { for } x \in D_{\nu}, \nu=1,2 \text {. }
$$


By combining (3.2) and (3.3) one obtains

$$
d\left(z, \partial D_{1}\right) \lesssim\left|\psi_{1}(z)\right|=\left|\varphi_{2}(F(z))\right| \lesssim d\left(F(z), \partial D_{2}\right)^{1 / l}
$$

and

$$
d\left(F(z), \partial D_{2}\right) \lesssim\left|\gamma_{2}(F(z))\right| \leqq\left|\varphi_{1}(z)\right| \lesssim d\left(z, \partial D_{1}\right)^{1 / l}
$$

Theorem 3.4. Let $D_{1}$ and $D_{2}$ be bounded domains in $C^{n}$ with smooth boundary. Suppose there is $\delta>0$ such that the Carathéodory metric of $D_{2}$ satisfies

$$
C_{\nu_{2}}(w, v) \gtrsim|v| d\left(w, \partial D_{2}\right)^{-\hat{o}}
$$

for all $w \in D_{2}$ and $v \in C^{n}$. Then there is $\alpha>0$, such that every proper holomorphic map $F: D_{1} \rightarrow D_{2}$ is Hölder continuous of order $\alpha$, i.e., there is $K<\infty$, such that

$$
\left|F(z)-F\left(z^{*}\right)\right| \leqq K\left|z-z^{*}\right|^{\alpha} \text { for all } z, z^{*} \in D_{1} .
$$

Proof. The hypothesis (*) implies that $D_{2}$ is a domain of holomorphy, hence pseudoconvex. If there is a proper holomorphic map $F: D_{1} \rightarrow D_{2}$, then $D_{1}$ must be pseudoconvex also, and hence 3.1 holds. So one has all the ingredients which are required to apply the classical argument of Henkin and Pinchuk. As the argument is very short, we include it here for the convenience of the reader.

By applying $\left(^{*}\right)$ to $F(z)$ and $F_{*_{z}} v$, and by Lemma 2.1, one obtains

$$
F_{* z} v\left|d\left(F(z), \partial D_{2}\right)^{-\hat{o}} \lesssim C_{D_{2}}\left(F(z), F_{*_{z}} v\right) \leqq C_{D_{1}}(z, v) \leqq\right| v \mid d\left(z, \partial D_{1}\right)^{-1} ;
$$

by multiplying with $d\left(F(z), \partial D_{2}\right)^{\tilde{a}}$ and 3.1 ,

$$
\left|F_{* z} v\right| \lesssim|v| d\left(z, \partial D_{1}\right)^{-1+\partial / l} \text {, }
$$

i.e.,

$$
\left\|F_{*_{z}}\right\| \lesssim d\left(z, \partial D_{1}\right)^{-1+\alpha}, \text { with } \alpha=\delta / l>0 .
$$

The analogue of a classical result of Hardy and Littlewood now implies that $F$ is Hölder continuous of order $\alpha$.

Theorem 2.2. and Theorem 3.4 clearly imply the Main Theorem stated in the introduction. From Corollary 1.13 one obtains the following special case of the Main Theorem.

Corollary 3.5. Let $D_{1}$ and $D_{2}$ be bounded convex domains in $C^{n}$ with real analytic boundary. Then every biholomorphic map $F: D_{1} \rightarrow D_{2}$ extends to a homeomorphism $\hat{F}: \bar{D}_{1} \rightarrow \bar{D}_{2}$. 
Open Problems 3.6. Some natural questions arise at this point. First, one would expect that the extension $\hat{F}$ in 3.5 is differentiable, or even real analytic, up to the boundary. Next, one may ask whether Corollary 3.5 remains true if one only assumes that $D_{1}$ and $D_{2}$ are bounded pseudoconvex domains with smooth boundary. Finally, one may consider similar questions for proper holomorphic maps; specifically, can the Main Theorem be strengthened to yield a differentiable extension to the boundary? It appears that methods quite different from those used in this paper would be needed to attack any of these problems.

\section{REFERENCES}

1. H. Behnke and P. Thullen, Theorie der Funktionen mehrerer komplexer Veränderlichen, 2nd rev. ed., Springer-Verlag, Berlin-Heidelberg-New York, 1970.

2. L. Bers, F. John and M. Schechter, Partial Differential Equations. Interscience, New York, 1966.

3. K. Diederich, and J. E. Fornaess, Exhaustion functions and Stein neighborhoods for smooth pseudoconvex domains. Proc. Nat. Acad. Sci. U.S.A., 72 (1975), 3279-3280. 4. - A strange bounded smooth domain of holomorphy, Bull. Amer. Math. Soc., 82 (1976), 74-76.

5. C. Fefferman, The Bergman kernel and biholomorphic mappings of pseudoconvex domains, Invent. Math., 26 (1974), 1-65.

6. I. Graham, Boundary behavior of the Carathéodory and Kobayashi metrics on strongly pseudoconvex domains in $C^{n}$ with smooth boundary, Trans. Amer. Math. Soc., 207 (1975), 219-240.

7. R. C. Gunning and H. Rossi, Analytic Functions of Several Complex Variables, Prentice-Hall, Englewood Cliffs, N.J., 1965.

8. M. Hakim and N. Sibony, Frontière de Silov et spectre de $A(D)$ pour des domaines faiblement pseudoconvexes, C. R. Acad. Sci. Paris, 281 (1975), A959-A962.

9. G. M. Henkin, An analytic polyhedron is not holomorphically equivalent to a strictly pseudoconvex domain, Dokl. Akad. Nauk SSSR, 210 (1973), 1026-1029=Soviet Math. Dokl., 14 (1973), 858-862.

10. L. Hörmander, $L^{2}$ estimates and existence theorems for the $\bar{\partial}$ operator, Acta Math., 113 (1965), 89-152.

11. M. Keldyš and M. Lavrent'ev, Sur l'unicité de la solution du problème de Neumann, C. R. (Dokl.) Acad. Sci. URSS, 26 (1937), 141-142.

12. J. J. Kohn, Global regularity for $\bar{\partial}$ on weakly pseudoconvex manifolds, Trans. Amer. Math. Soc., 181 (1973), 273-292.

13. J. J. Kohn and L. Nirenberg, A pseudoconvex domain not admitting a holomorphic support function, Math. Ann., 201 (1973), 265-268.

14. S. Lojasiewicz, Sur le problème de la division, Studia Math., 18 (1959), 87-136.

15. P. Pflug, Über polynomiale Funktionen auf Holomorphiegebieten, Math. Z., 139 (1974), 133-139.

16. S. J. Pinchuk, On proper holomorphic mappings of strictly pseudoconvex domains, Siberian Math. J., 15 (1975), 644-649.

17. R. M. Range, On the topological extension to the boundary of biholomorphic maps in $C^{n}$, Trans. Amer. Math. Soc., 216 (1976), 203-216.

18. - Holder estimates for $\bar{\partial}$ on convex domains in $\boldsymbol{C}^{2}$ with real analytic boundary, Proc. Symp. Pure Math., 30, part 2, 31-33. Amer. Math. Soc., Providence, R. I., 1977. 
19. H. Rossi, Holomorphically convex sets in several complex variables, Ann. Math., 74 (1961), 470-493.

20. N. Vormoor, Topologische Fortsetzung biholomorpher Funktionen auf dem Rande bei beschränkten streng pseudokonvexen Gebieten im $C^{n}$ mit $C^{\infty}$-Rand, Math. Ann., 204 (1973), 239-261.

Received September 29, 1977. Research supported by NSF Grant MCS75-07062A01.

State University of New York

ALBANY, NY 12222 



\section{PACIFIC JOURNAL OF MATHEMATICS}

\section{EDITORS}

RICHARD ARENS (Managing Editor)

University of California

Los Angeles, California 90024

C. W. CurTis

University of Oregon

Eugene, OR 97403

C. C. MOORE

University of California

Berkeley, CA 94720
J. DUGUNDJI

Department of Mathematics University of Southern California Los Angeles, California 90007

R. FinN AND J. Milgram Stanford University Stanford, California 94305

\section{ASSOCIATE EDITORS}

E. F. BECKENBACH

B. H. NeumanN

F. WOLF

K. YoSHIDA

\section{SUPPORTING INSTITUTIONS}

UNIVERSITY OF BRITISH COLUMBIA CALIFORNIA INSTITUTE OF TECHNOLOGY UNIVERSITY OF CALIFORNIA MONTANA STATE UNIVERSITY UNIVERSITY OF NEVADA, RENO NEW MEXICO STATE UNIVERSITY OREGON STATE UNIVERSITY UNIVERSITY OF OREGON
UNIVERSITY OF SOUTHERN CALIFORNIA

STANFORD UNIVERSITY

UNIVERSITY OF HAWAII

UNIVERSITY OF TOKYO

UNIVERSITY OF UTAH

WASHINGTON STATE UNIVERSITY

UNIVERSITY OF WASHINGTON 


\section{Pacific Journal of Mathematics \\ Vol. 78, No. $1 \quad$ March, 1978}

Simeon M. Berman, A class of isotropic distributions in $\mathbf{R}^{n}$ and their

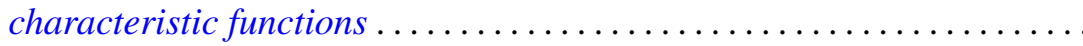

Ezra Brown and Charles John Parry, The 2-class group of biquadratic fields.

II ........................................ 11

Thomas E. Cecil and Patrick J. Ryan, Focal sets of submanifolds ....... 27

Joseph A. Cima and James Warren Roberts, Denting points in $B^{p} \ldots \ldots \ldots 41$

Thomas W. Cusick, Integer multiples of periodic continued fractions . . . . . 47

Robert D. Davis, The factors of the ramification sequence of a class of

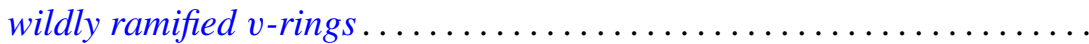

Robert Martin Ephraim, Multiplicative linear functionals of Stein

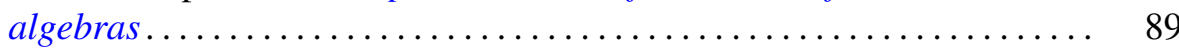

Philip Joel Feinsilver, Operator calculus . .................... 95

David Andrew Gay and William Yslas Vélez, On the degree of the splitting field of an irreducible binomial ..........................

Robert William Gilmer, Jr. and William James Heinzer, On the divisors of

monic polynomials over a commutative ring ..................

Robert E. Hartwig, Schur's theorem and the Drazin inverse .............

Hugh M. Hilden, Embeddings and branched covering spaces for three and four dimensional manifolds ............................

Carlos Moreno, The Petersson inner product and the residue of an Euler product. ...

Christopher Lloyd Morgan, On relations for representations of finite groups....

Ira J. Papick, Finite type extensions and coherence

$\mathrm{R}$. Michael Range, The Carathéodory metric and holomorphic maps on a class of weakly pseudoconvex domains ................

Donald Michael Redmond, Mean value theorems for a class of Dirichlet series

Daniel Reich, Partitioning integers using a finitely generated semigroup ...

Georg Johann Rieger, Remark on a paper of Stux concerning squarefree

numbers in non-linear sequences

Gerhard Rosenberger, Alternierende Produkte in freien Gruppen ..

Ryōtarō Satō, Contraction semigroups in Lebesgue space

Tord Sjödin, Capacities of compact sets in linear subspaces of $\mathbf{R}^{n}$

Robert Jeffrey Zimmer, Uniform subgroups and ergodic actions of exponential Lie groups......................... 